\title{
Distribuição espacial de fósforo em Latossolo tratado com lodo de esgoto e adubação mineral
}

\author{
Vanessa L. Costa ${ }^{1}$, Isabella C. de Maria ${ }^{2}$, Otávio A. Camargo ${ }^{3}$, Célia R. Grego ${ }^{4}$ \& Leônidas C. A. Melo \\ ${ }^{1}$ IAC. Campinas, SP. E-mail: van_leca@yahoo.com.br \\ ${ }^{2}$ IAC. Campinas, SP. E-mail: icdmaria@iac.sp.gov.br. Bolsista em produtividade do CNPq. (Autor correspondente) \\ ${ }^{3}$ IAC. Campinas, SP. E-mail: ocamargo@iac.sp.gov.br. Bolsista em produtividade do CNPq \\ ${ }^{4}$ Embrapa Monitoramento por Satélite. Campinas, SP. E-mail: celia.grego@embrapa.br \\ ${ }^{5}$ UFV. Viçosa, MG. E-mail: leonidas.melo@ufv.br
}

\section{Palavras-chave:}

resíduo orgânico

erosão de solo

fertilização

\begin{abstract}
R E S U M O
Em solos agrícolas que recebem aplicação de lodo de esgoto pode ocorrer, quando sujeitos à erosão hídrica, intenso transporte de fósforo $(\mathrm{P})$ para trechos inferiores da paisagem e, eventualmente, para os corpos de água. O objetivo com este trabalho foi avaliar a distribuição espacial de P em um Latossolo Vermelho eutroférrico, cultivado com milho. Os tratamentos foram avaliados com aplicação de adubo mineral (AM) e de lodo de esgoto (LE). Amostras de solo foram coletadas seguindo uma grade amostral de 69 pontos em cada tratamento, em duas épocas, no início e no final do ciclo da cultura, em uma área experimental com $10 \%$ de declividade. No tratamento AM houve dependência espacial moderada de $\mathrm{P}$ no solo ocorrendo maiores concentrações de $\mathrm{P}$ na parte inferior do talhão, causada pela erosão do solo. O mesmo padrão foi observado para soma de bases, capacidade de troca catiônica e matéria orgânica do solo. Por outro lado, a aplicação de LE, feita de maneira não uniforme, resulta em sítios com maiores teores de fósforo aleatoriamente distribuídos e correlacionados com a matéria orgânica do solo.
\end{abstract}

\section{Key words:} organic residue soil erosion fertilization

\section{Spatial distribution of phosphorus in an Oxisol amended with sewage sludge and mineral fertilization}

\begin{abstract}
A B S T R A C T
In agricultural soils receiving sewage sludge and subjected to water erosion, may occur intense transport of phosphorus (P) to the lower parts of the landscape and eventually to water bodies. The objective of this study was to evaluate the spatial distribution of $\mathrm{P}$ in an Oxisol cultivated with maize. The treatments were evaluated with application of mineral fertilization (MF) and sewage sludge (SS). Soil samples were collected following a sampling grid of 69 points in each treatment at the beginning and at the end of the crop cycle, in an experimental area with $10 \%$ slope. There was a moderate spatial dependence of $\mathrm{P}$ in the soil in the treatment of MF, resulting in higher concentrations of $\mathrm{P}$ in the deeper layers of the soil caused by erosion. The same standard was observed for the soil attributes sum of bases, cation exchange capacity and soil organic matter. Conversely, when SS was applied there was a randomized distribution of $\mathrm{P}$ in the plots because of the non-uniform manner of its application. Such random distribution of $\mathrm{P}$ showed correlation with soil organic matter.
\end{abstract}

\section{INTRODUÇÃO}

Uma característica marcante do fósforo (P) é sua baixa disponibilidade em decorrência da baixa difusão e da alta fixação em solos (Shen et al., 2011). Para atender à demanda das culturas de alta produção a adição de $\mathrm{P}$, seja ela via fertilizantes minerais ou orgânicos, tornou-se uma prática indispensável na maioria dos solos brasileiros. A aplicação de lodo de esgoto no solo pode resultar em aumento dos teores de $\mathrm{P}$, tanto associado às partículas do solo ou às partículas do próprio lodo, como demonstrado em trabalhos já realizados em diferentes tipos de solo (Bettiol \& Ghini, 2011). O acúmulo de P, resultante da quantidade aplicada excessivamente àquela removida pelas culturas e por longos períodos, contribui para aumentar o potencial de perda de P para o ambiente (Elliott \& O'Connor, 2007).

Em solos agrícolas sujeitos à ação erosiva das chuvas e às variações na cobertura dos solos, de acordo com o desenvolvimento das culturas, pode ocorrer o arraste desse elemento por erosão para posições inferiores da paisagem atingindo os corpos de água. Em condições propícias as frações de P, inorgânica e orgânica, tanto nas formas dissolvidas como particulada, são passíveis de perda para o ambiente aquático. As concentrações das formas de $\mathrm{P}$ transportadas variam $\mathrm{e}$ dependem, dentre outros fatores, da dose e das características químicas do lodo e dos fertilizantes aplicados. Esta transferência 
é conduzida via escoamento superficial pela ação do processo de erosão em virtude da interação da água da chuva com a camada superficial do solo (Mori et al., 2009). Entre as formas de P perdidas via enxurrada, as particuladas e orgânicas apresentam maiores riscos de degradação dos corpos de água por agirem como fonte de $\mathrm{P}$ em longo prazo (Hart et al., 2004), ao passo que as dissolvidas são rapidamente biodisponíveis.

É importante frisar que perdas de P relativamente pequenas podem causar sérias consequências ambientais visto que sua presença, mesmo que em quantidades módicas nas águas naturais, pode impulsionar o processo de eutrofização (Hart et al., 2004), já que o P é considerado um nutriente limitante em ambientes aquáticos. Para o setor agrícola essas perdas podem não apresentar importância econômica mas promovem impactos indiretos negativos, visto que, em sendo difusa, a contaminação proveniente de áreas agrícolas pode ocorrer a quilômetros de distância da fonte (Haygarth \& Jarvis, 1999).

A redistribuição do fósforo e de outros elementos do solo, decorrente do arraste para outras posições da paisagem, pode ser investigada pela análise de variabilidade espacial. Nos solos é comum a existência de atributos que apresentam dependência espacial e a geoestatística tem as ferramentas para analisar esses dados, desde que se tenha as coordenadas geográficas da posição de onde as amostras foram retiradas (Vieira, 2000). Entre os produtos da análise geoestatística podem ser construídos mapas que mostram o comportamento da distribuição espacial de cada atributo do solo e comparar seu comportamento e relação na paisagem (Camargo et al., 2012).

O objetivo com este trabalho foi avaliar a distribuição espacial do $\mathrm{P}$ e dos atributos químicos $\mathrm{pH}, \mathrm{MO}$, SB e CTC, num Latossolo Vermelho eutroférrico cultivado com milho, em parcelas sujeitas à ação da erosão hídrica, comparando-se os tratamentos AM (com adubação mineral) e LE (com aplicação anual de lodo de esgoto), sendo as amostras de solo coletadas nos períodos inicial e final do ciclo de uma cultura de milho.

\section{MAterial e Métodos}

O solo da área experimental é um Latossolo Vermelho eutroférrico de textura argilosa (EMBRAPA, 2006). Os valores dos atributos físicos e químicos na camada $0-0,20 \mathrm{~m}$, antes do início do experimento, são apresentados na Tabela 1.

O clima da região, de acordo com a classificação de Köppen, é caracterizado como tropical úmido e apresenta duas estações distintas: chuvosa no verão e seca no inverno, com valores médios anuais de $20,5^{\circ} \mathrm{C}$ para a temperatura e $1.400 \mathrm{~mm}$ para precipitação. O período chuvoso ocorre entre os meses de outubro e março, correspondente a $76 \%$ do total das chuvas.

O experimento foi instalado no campo, em dezembro de 2001. A área experimental, utilizada para estudos de erosão hídrica, é composta por parcelas de 4 × 25 m $\left(100 \mathrm{~m}^{2}\right)$ com $10 \%$ de declividade.

Para esse estudo foram utilizadas duas parcelas: a) uma tratada com lodo na quantidade suficiente para suprir, de duas vezes, a dose necessária de $\mathrm{N}$ para a cultura de milho $\left(20 \mathrm{Mg} \mathrm{ha}^{-1}\right.$ de lodo de esgoto por ano) e adubação complementar de $17 \mathrm{~kg}$ ha $^{-1}$ de $_{2} \mathrm{O}$, denominada LE e b) outra com adubação mineral, recebendo um formulado N-P-K (4,8 kg ha-1 de N , $48 \mathrm{~kg} \mathrm{ha}^{-1} \mathrm{de}$ $\mathrm{P}_{2} \mathrm{O}_{5}, 17 \mathrm{~kg} \mathrm{ha}^{-1}$ de $\mathrm{K}_{2} \mathrm{O}$ ) e $165 \mathrm{~kg} \mathrm{ha}^{-1}$ de $\mathrm{N}$ via ureia, por ano, denominada AM. A determinação da dose de adubo mineral teve, como base, a análise do solo e recomendações técnicas para a cultura do milho, de acordo com Raij et al. (1996). A dose de lodo de esgoto foi determinada como a quantidade de lodo suficiente para suprir, em duas vezes, a dose necessária de $\mathrm{N}$ para a cultura de milho, a qual foi definida em função da análise química do lodo e da necessidade de nitrogênio do milho, utilizando adubação complementar com $\mathrm{KCl}$, devido à deficiência deste elemento no lodo de esgoto utilizado. A quantidade média de $\mathrm{P}$ adicionada pelo lodo foi de $376 \mathrm{~kg} \mathrm{ha}^{-1} \mathrm{de}$ $\mathrm{P}_{2} \mathrm{O}_{5}$ por ano; em novembro de 2004 foram aplicados $4 \mathrm{Mg} \mathrm{ha}^{-1}$ de calcário dolomítico para elevar o $\mathrm{pH}$ do solo.

O lodo utilizado foi procedente da Estação de Tratamento de Esgoto (ETE) de Jundiaí, SP, cujo efluente foi tratado biologicamente, sendo a remoção da carga orgânica realizada em duas etapas subsequentes: digestão aeróbia e estabilização anaeróbia em lagoas de decantação. Ao lodo gerado foram adicionados polímeros com a finalidade de otimizar a floculação do material que foi, então, desaguado e acondicionado em leitos de secagem. O valor médio do $\mathrm{pH}\left(\mathrm{H}_{2} \mathrm{O}\right)$ foi de 7,9 e os teores de metais pesados (Tabela 2) se encontraram abaixo da concentração máxima permitida pela norma P.4230 da CETESB (1999).

A disposição e a incorporação do lodo no solo foram feitas manualmente, na camada $0,10 \mathrm{~m}$, com o auxílio de enxada, anualmente, de 2001 a 2006, entre 5 a 10 dias antes da semeadura do milho. A cultura do milho (IAC 21) foi plantada em contorno

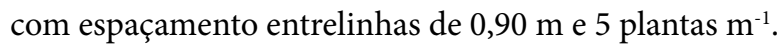

Foram feitas duas amostragens da camada 0-0,05 m: uma em dezembro de 2006 ( $1^{\text {a }}$ época, 30 dias após a aplicação do lodo) e outra em maio de 2007 ( $2^{a}$ época, ao final do período que concentra a maior parte das chuvas na região). As amostras foram coletadas em 69 pontos georreferenciados dentro de cada parcela, sendo 21 amostras na parte superior e 21 na parte inferior, com espaçamento de $1,0 \times 1,0 \mathrm{~m}$ e 27 amostras na parte intermediária, com 21 amostras espaçadas $1,0 \times 1,0 \mathrm{~m}$ intercaladas com mais oito espaçadas $1,5 \times 1,5 \mathrm{~m}$.

As amostras de solo foram secadas em estufa de circulação forçada de ar a temperatura de $30^{\circ} \mathrm{C}$ e, posteriormente, passadas por peneira de malha de $2 \mathrm{~mm}$. Os teores de $\mathrm{P}$ foram extraídos

Tabela 1. Características físicas e químicas do Latossolo Vermelho eutroférrico, antes do início do ensaio, na camada de $0-0,20 \mathrm{~m}$

\begin{tabular}{|c|c|c|c|c|c|c|c|c|c|c|c|c|c|}
\hline Areia & Silte & Argila & \multirow{2}{*}{$\underset{\mathrm{pH}}{\mathrm{paCl}{ }_{2}}$} & \multirow{2}{*}{$\begin{array}{l}\text { P-Resina } \\
\mathrm{mg} \mathrm{dm}^{-3}\end{array}$} & $\mathrm{Ca}$ & $\mathrm{Mg}$ & $\mathbf{K}$ & $\mathrm{H}+\mathrm{Al}$ & $T$ & $S$ & \multirow{2}{*}{$\begin{array}{l}\mathbf{V} \\
\%\end{array}$} & \multirow{2}{*}{$\begin{array}{c}\mathrm{MO} \\
\mathrm{g} \mathrm{dm}^{-3}\end{array}$} & \multirow{2}{*}{$\begin{array}{c}\mathrm{Fe} \\
\mathrm{mg} \mathrm{dm}\end{array}$} \\
\hline & $\mathrm{g} \mathrm{kg}$ & & & & \multicolumn{6}{|c|}{$\mathrm{mmol}_{\mathrm{c}} \mathrm{dm}^{-3}$} & & & \\
\hline 287 & 132 & 581 & 4,6 & 22,7 & 10,6 & 3,3 & 2,6 & 45,5 & 62,1 & 16,6 & 27,4 & 20,7 & 21,3 \\
\hline
\end{tabular}

Análise granulométrica de acordo com Camargo et al. (1986) e parâmetros da fertilidade do solo de acordo com Raij et al. (2001) 
Tabela 2. Composição média dos lodos de esgoto aplicados anualmente no experimento entre 2001 e 2006

\begin{tabular}{lc}
\hline \multicolumn{1}{c}{ Parâmetro(1,2) } & Valor \\
pH & 7,9 \\
Umidade (\%) & 64,6 \\
Sólidos voláteis (\%) & 57,1 \\
C orgânico $\left(\mathrm{g} \mathrm{kg}^{-1}\right)$ & 274 \\
Nitrogênio Kjeldahl $\left(\mathrm{g} \mathrm{kg}^{-1}\right)$ & 29,3 \\
Arsênio $\left(\mathrm{mg} \mathrm{kg}^{-1}\right)$ & $<0,01^{(3)}$ \\
Boro $\left(\mathrm{mg} \mathrm{kg}^{-1}\right)$ & 231 \\
Cádmio $\left(\mathrm{mg} \mathrm{kg}^{-1}\right)$ & 9,0 \\
Cálcio $\left(\mathrm{g} \mathrm{kg}^{-1}\right)$ & 15,7 \\
Chumbo $\left(\mathrm{mg} \mathrm{kg}^{-1}\right)$ & 168 \\
Cobre $\left(\mathrm{mg} \mathrm{kg}^{-1}\right)$ & 562 \\
Cromo total $\left(\mathrm{mg} \mathrm{kg}^{-1}\right)$ & 161 \\
Enxofre $\left(\mathrm{g} \mathrm{kg}^{-1}\right)$ & 20,2 \\
Ferro $\left(\mathrm{g} \mathrm{kg}^{-1}\right)$ & 22,4 \\
Fósforo $\left(\mathrm{g} \mathrm{kg}^{-1}\right)$ & 8,2 \\
Magnésio $\left(\mathrm{g} \mathrm{kg}^{-1}\right)$ & 1,2 \\
Mercúrio $\left(\mathrm{mg} \mathrm{kg}^{-1}\right)$ & $<0,01^{(3)}$ \\
Níquel $\left(\mathrm{mg} \mathrm{kg}^{-1}\right)$ & 37,1 \\
Selênio $\left(\mathrm{mg} \mathrm{kg}^{-1}\right)$ & $<0,01^{(3)}$ \\
Zinco $\left(\mathrm{mg} \mathrm{kg}^{-1}\right)$ & 1321 \\
Potássio $\left(\mathrm{g} \mathrm{kg}^{-1}\right)$ & 2,8 \\
Bário $\left(\mathrm{mg} \mathrm{kg}^{-1}\right)$ & 235 \\
\hline
\end{tabular}

${ }^{1}$ Valores de concentração são dados com base na matéria seca.

${ }^{2}$ Determinação do $\mathrm{C}$ orgânico por titulometria com digestão com dicromato em bloco digestor (Raij et al., 2001). Para metais e $P$ foram seguidas as orientações de USEPA, método 3051. Umidade e sólidos voláteis determinados por perda de massa a 60 e $500^{\circ} \mathrm{C}$, respectivamente. Determinou-se o pH em extrato aquoso (1:5), descritos em Raij et al. (2001).

${ }^{3}$ Não detectado

pelo método da Resina Trocadora de Ânions (Raij et al., 2001) e por Mehlich I (Silva, 1999) e só então determinados por colorimetria em espectrofotômetro a $800 \mathrm{~nm}$. Foram avaliados $\mathrm{pH}$ em $\mathrm{CaCl}_{2}$ e matéria orgânica (MO) pelo dicromato de potássio (Raij et al., 2001) e calculados os valores de soma de bases (SB) e capacidade de troca catiônica (CTC).

Os dados foram analisados por meio de parâmetros estatísticos descritivos (média, variância, coeficiente de variação, assimetria, curtose) e de correlação entre as variáveis analisadas. Para avaliar a normalidade dos dados foi utilizado o teste Kolmogorov-Sminorv. Levando-se em consideração as coordenadas geográficas dos pontos amostrados foram calculados os semivariogramas para cada variável seguindo a metodologia de Vieira (2000). Para as variáveis que apresentaram dependência espacial foi ajustado um modelo de semivariograma e calculada a krigagem ordinária para estimar valores em qualquer posição dentro do campo, sem tendência e com variância mínima. Para as variáveis que apresentaram tendência a avaliação da dependência espacial foi feita com resíduos obtidos pela diferença entre a superfície de tendência e os valores amostrados (Vieira et al., 2002). A partir dos dados estimados por krigagem, mapas de isolinhas foram gerados no programa SURFER 7.0.

\section{Resultados E Discussão}

O tratamento LE resultou em aumento dos teores de $\mathrm{P}$, de matéria orgânica (MO) e da capacidade de troca catiônica (CTC) e redução nos valores de soma de bases (SB) e pH do solo nas duas épocas de amostragem, em comparação ao tratamento AM (Tabela 3).

Os altos valores de P-Resina e P-Mehlich I encontrados no tratamento LE reforçam que, se praticada continuamente, a aplicação de lodo conduz ao acúmulo de P lábil na superfície do solo, concordando com resultados de outros autores (Kidd et al., 2007; Trannim et al., 2008).

A acidificação do solo tratado com lodo também foi observada por Boeira et al. (2007) e Trannim et al. (2008), os quais utilizaram lodos condicionados sem a adição de cal. Lodos de esgoto que não recebem adição de cal têm provocado decréscimo dos valores de $\mathrm{pH}$ já nas primeiras aplicações. Isto se deve à dissociação de prótons ligados aos grupos funcionais $\mathrm{COOH}$ e $\mathrm{OH}$ da matéria orgânica, além da decomposição do lodo pela atividade microbiana, que tem sido apontada como a principal causa da acidificação dos solos visto que a mineralização do C, $\mathrm{N}$ e S libera prótons para o meio (Souza et al., 2007a).

Da primeira para segunda época de amostragem observouse, nos dois tratamentos, redução dos valores de MO, SB, CTC e pH (Tabela 3) provavelmente pela mineralização da matéria orgânica dos resíduos culturais e do lodo de esgoto, pela

Tabela 3. Parâmetros da estatística descritiva para os atributos químicos do solo nos tratamentos adubação mineral (AM) e lodo de esgoto (LE) no início do desenvolvimento da cultura do milho ( $1^{\text {a }}$ época) e após a colheita ( $2^{\mathrm{a}}$ época)

\begin{tabular}{|c|c|c|c|c|c|c|c|}
\hline Variável & $M$ & Min-Máx & $\mathbf{V}$ & CV & $\bar{A}$ & C & D \\
\hline & \multicolumn{7}{|c|}{$\begin{array}{c}1^{\text {a }} \text { Época } \\
\text { AM }\end{array}$} \\
\hline P-Mehlich I (mg dm-3) & 28 & $4-83$ & 421 & 74,0 & 0,94 & $-0,45$ & 0,119 \\
\hline P-Resina $\left(\mathrm{mg} \mathrm{dm}^{-3}\right)$ & 60 & $18-153$ & 644 & 42,0 & 1,51 & 3,38 & 0,156 \\
\hline $\mathrm{MO}\left(\mathrm{g} \mathrm{dm}^{-3}\right)$ & 26 & $22-29$ & 2 & 6,0 & 0,12 & 0,01 & 0,222 \\
\hline $\mathrm{SB}\left(\mathrm{mmol}_{\mathrm{c}} \mathrm{dm}^{-3}\right)$ & 80,5 & $33,5-148,7$ & 470,2 & 26,9 & 0,74 & 0,56 & 0,098 \\
\hline CTC $\left(\mathrm{mmol}_{\mathrm{c}} \mathrm{dm}^{-3}\right)$ & 101,2 & $64,3-163,5$ & 373,4 & 19,0 & 1,04 & 1,05 & 0,156 \\
\hline $\mathrm{pH}$ & 6 & $5-6,6$ & 0,1 & 5,0 & $-0,75$ & 0,37 & 0,460 \\
\hline P-Mehlich I (mg dm-3) & 84 & $30-168$ & 566 & 28,0 & 0,65 & 1,37 & 0,113 \\
\hline P-Resina $\left(\mathrm{mg} \mathrm{dm}^{-3}\right)$ & 133 & 60-262 & 2137 & 35,0 & 0,46 & $-0,30$ & 0,076 \\
\hline $\mathrm{MO}\left(\mathrm{g} \mathrm{dm}^{-3}\right)$ & 47 & $32-70$ & 52 & 15,0 & 0,30 & 0,56 & 0,103 \\
\hline $\mathrm{SB}\left(\mathrm{mmol}_{\mathrm{c}} \mathrm{dm}^{-3}\right)$ & 35,7 & $16,5-83,6$ & 134,3 & 32,5 & 1,17 & 3,01 & 0,157 \\
\hline CTC $\left(\mathrm{mmol}_{\mathrm{c}} \mathrm{dm}^{-3}\right)$ & 140,4 & $106,6-180,9$ & 207,8 & 10,3 & $-0,01$ & 0,17 & 0,079 \\
\hline \multirow[t]{2}{*}{$\mathrm{pH}$} & 4 & $3,7-5$ & 0 & 4,0 & 1,75 & 7,91 & 0,530 \\
\hline & \multicolumn{7}{|c|}{ 2a Época } \\
\hline P-Mehlich I (mg dm-3) & 42 & $20-97$ & 334 & 43,0 & 1,22 & 0,95 & 0,162 \\
\hline P-Resina (mg dm ${ }^{-3}$ ) & 57 & $23-119$ & 566 & 42,0 & 0,82 & 0,03 & 0,111 \\
\hline $\mathrm{MO}\left(\mathrm{g} \mathrm{dm}^{-3}\right)$ & 23 & $20-28$ & 2 & 6,0 & 0,42 & $-0,12$ & 0,151 \\
\hline $\mathrm{SB}\left(\mathrm{mmol}_{\mathrm{c}} \mathrm{dm}^{-3}\right)$ & 67,1 & $35,5-121,5$ & 266,3 & 24,3 & 0,65 & 0,75 & 0,091 \\
\hline CTC $\left(\mathrm{mmol}_{\mathrm{c}} \mathrm{dm}^{-3}\right)$ & 84,7 & $58,6-133,5$ & 210,1 & 17,1 & 0,78 & 0,75 & 0,092 \\
\hline $\mathrm{pH}$ & 6 & $5,1-6,4$ & 0 & 4,0 & $-1,34$ & 2,57 & 0,539 \\
\hline P-Mehlich I (mg dm-3) & 114 & 48-203 & 1040 & 28,0 & 0,24 & 0,19 & 0,067 \\
\hline P-Resina (mg dm-3) & 163 & $64-356$ & 3348 & 36,0 & 0,94 & 1,61 & 0,114 \\
\hline $\mathrm{MO}\left(\mathrm{g} \mathrm{dm}^{-3}\right)$ & 40 & $26-53$ & 25 & 13,0 & $-0,03$ & 0,61 & 0,104 \\
\hline $\mathrm{SB}\left(\mathrm{mmol}_{\mathrm{c}} \mathrm{dm}^{-3}\right)$ & 26,5 & $8,7-63,1$ & 102 & 38,0 & 0,96 & 1,49 & 0,089 \\
\hline CTC $\left(\mathrm{mmol}_{\mathrm{c}} \mathrm{dm}^{-3}\right)$ & 134,9 & $87,5-175,6$ & 350,5 & 13,9 & $-0,31$ & $-0,44$ & 0,101 \\
\hline $\mathrm{pH}$ & 4 & $3,7-4,5$ & 0 & 4,0 & 0,68 & 0,41 & 0,530 \\
\hline
\end{tabular}


absorção pela cultura do milho e pelo arraste por lixiviação ou enxurrada dos nutrientes catiônicos. $\mathrm{O}$ aumento nos teores de P-Mehlich I e P-resina para a segunda época no tratamento LE pode estar relacionado às altas concentrações de $\mathrm{P}$ do lodo que, com a decomposição da MO, foi liberado no solo.

O método Mehlich I extraiu menos P disponível em ambos os tratamentos em comparação com o método da Resina. Tal fato tem sido observado em solos que apresentam teores de argila superiores a $200 \mathrm{~g} \mathrm{~kg}^{-1}$ e altas concentrações de óxidos de ferro e de alumínio, características inerentes ao solo utilizado neste trabalho. Nesses solos o $\mathrm{SO}_{4}^{2-}$ do extrator Mehlich I, que atua por troca com os fosfatos adsorvidos, pode ter sido adsorvido aos sítios de adsorção que não foram ocupados pelo $P$. Além do mais, a capacidade tampão deste solo pode ter elevado o valor de $\mathrm{pH}$ inicial do extrator de 1,2 a valores próximos ao $\mathrm{pH}$ do solo perdendo, desta forma, sua capacidade de extração (Novais et al., 2007). Munhoz et al. (2011) obtiveram resultados semelhantes quanto aos métodos de extração de $\mathrm{P}$ em Latossolo argiloso distroférrico cultivado com milho e tratado com lodo de esgoto durante cinco anos.

A variabilidade dos valores das variáveis estudadas foi avaliada pelo coeficiente de variação (CV). No tratamento AM a variabilidade dos atributos $\mathrm{MO}$ e $\mathrm{pH}$ mostrou-se baixa, para a CTC foi moderada, para SB alta e muito alta para P-Resina e P-Mehlich I (Tabela 3). No tratamento LE a tendência foi semelhante sendo que o coeficiente de variação dos atributos P-Resina e P-Mehlich I foi menor não atingindo a classificação de variabilidade muito alta. Os valores de CV e também a diferença entre os valores extremos de mínimos e máximos, revelam que esses atributos do solo são heterogêneos, o que dificulta, em alguns casos, o ajuste dos modelos teóricos às semivariâncias calculadas uma vez que a média aritmética dos dados amostrais é adotada para a estimativa das concentrações em locais não amostrados (Vieira, 2000).

Os coeficientes de assimetria e de curtose (Tabela 3 ) indicaram que algumas das variáveis apresentaram não normalidade da distribuição de frequências. Isto ocorreu para os atributos P-Resina e CTC em AM e para SB e pH em LE na primeira época enquanto para P-Mehlich I e pH em AM ocorreu na segunda época. Para os demais atributos os coeficientes de assimetria e curtose ficaram próximos de uma distribuição normal. Entretanto, o teste de Kolmogorov-Sminorv (Tabela 3) indicou a não normalidade dos dados, o que levou à decisão de normalização dos dados, exceto para a variável $\mathrm{pH}$, para o cálculo dos semivariogramas, visto que distribuições de frequência com cauda muito alongada poderiam comprometer a interpolação dos dados pela krigagem (Souza et al., 2007b).

Correlações lineares e significativas entre o P disponível (Resina e Mehlich I) e alguns dos atributos do solo foram observados nos tratamentos LE e AM, nos dois períodos de amostragens. Porém, os coeficientes de correlações foram muito baixos, em geral, inferiores a 50\% (Tabela 4).

De modo geral, o P disponível esteve mais correlacionado com a matéria orgânica (MO) no tratamento LE do que no
Tabela 4. Valores de $\mathrm{r}^{2}$ obtidos pela correlação entre fósforo disponível $(\mathrm{P})$ e os atributos do solo nos tratamentos adubação mineral (AM) e lodo de esgoto (LE) no início do desenvolvimento da cultura do milho ( $1^{\text {a }}$ época) e após a colheita ( $2^{\mathrm{a}}$ época)

\begin{tabular}{|c|c|c|c|c|c|c|}
\hline \multirow{2}{*}{ Época } & \multirow{2}{*}{$\mathbf{P}$} & \multirow{2}{*}{ Tratamento } & \multirow{2}{*}{$\begin{array}{c}\mathrm{MO} \\
\mathrm{g} \mathrm{dm}^{-3}\end{array}$} & SB & CTC & \multirow{2}{*}{$\mathrm{pH}$} \\
\hline & & & & \multicolumn{2}{|c|}{$\mathrm{mmol}_{\mathrm{c}} \mathrm{dm}^{-3}$} & \\
\hline \multirow{2}{*}{$1^{a}$} & \multirow{4}{*}{ Resina } & AM & $0,32^{*}$ & $0,47^{*}$ & $0,51^{*}$ & 0,49 * \\
\hline & & LE & $0,74^{*}$ & $0,24^{*}$ & $0,35^{\star}$ & $-0,01$ \\
\hline \multirow{2}{*}{$2^{a}$} & & AM & 0,16 & $0,35^{*}$ & $0,37^{*}$ & 0,22 * \\
\hline & & LE & $0,62^{*}$ & $0,28^{*}$ & $0,42^{*}$ & $-0,01$ \\
\hline \multirow{2}{*}{$1^{\mathrm{a}}$} & \multirow{4}{*}{ Mehlich I } & AM & $0,34^{*}$ & 0,08 & 0,20 & $-0,06$ \\
\hline & & LE & $0,66^{*}$ & 0,19 & 0,28 * & $-0,04$ \\
\hline \multirow{2}{*}{$2^{a}$} & & AM & $-0,04$ & 0,17 & 0,17 & 0,14 \\
\hline & & LE & $0,52^{*}$ & 0,10 & 0,49 * & $-0,24^{*}$ \\
\hline
\end{tabular}

AM - Adubação mineral; LE - 20 Mg ha-1 de lodo de esgoto por ano; MO - Matéria orgânica; SB - Soma de bases. CTC - Capacidade de troca catiônica.

* Correlação significativa a 0,05 de probabilidade

tratamento AM, principalmente na primeira época. De fato, esta correlação pôde ser observada pela similaridade de áreas com teores mais elevados dessas duas variáveis nos mapas de distribuição espacial (Figuras 1 e 2).

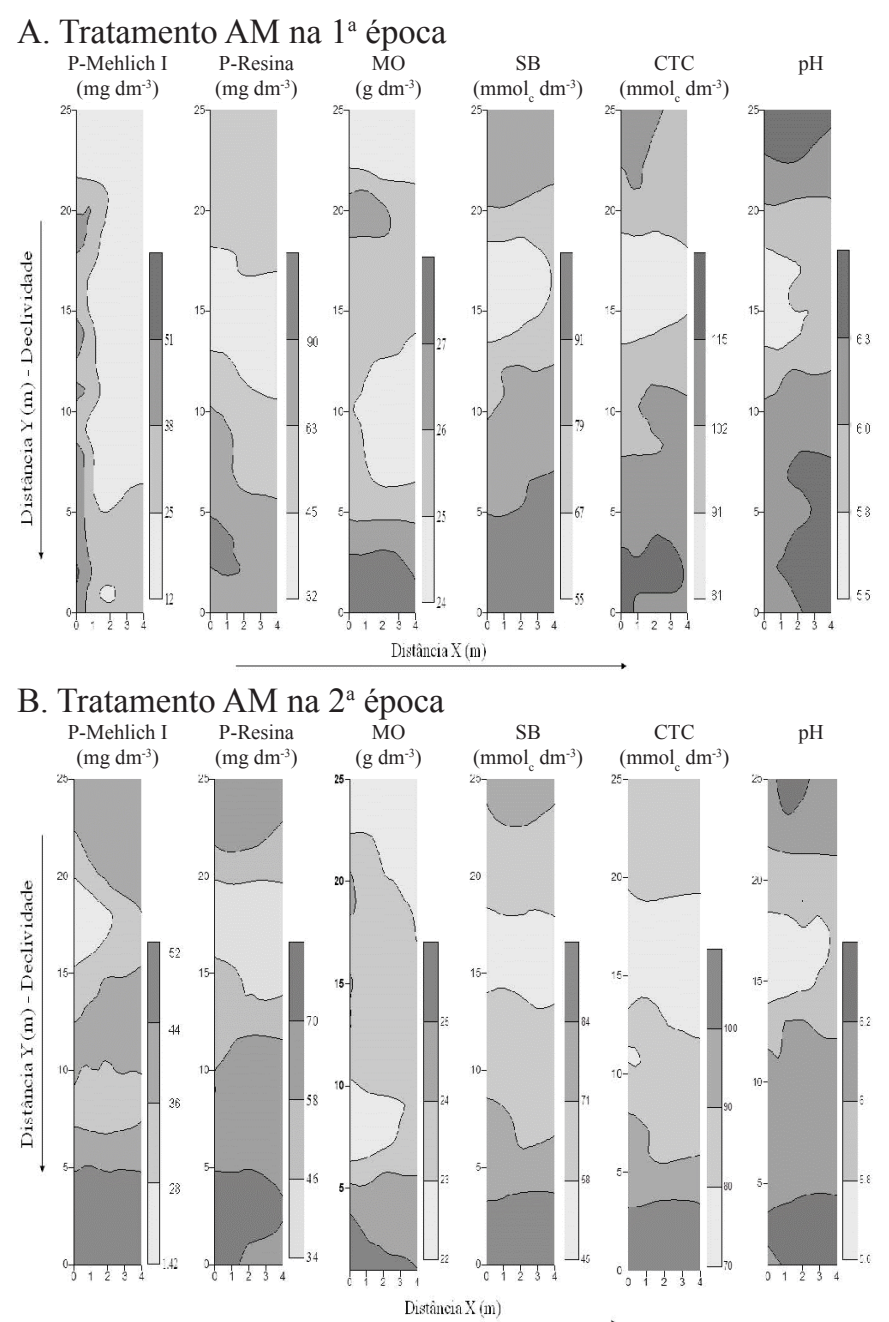

Figura 1. Mapas dos atributos químicos do solo para o tratamento adubação mineral (AM) na $1^{\mathrm{a}}(\mathrm{A})$ e $2^{\mathrm{a}}(\mathrm{B})$ épocas estudadas. MO - Matéria orgânica. SB - Soma de bases. CTC - Capacidade de troca catiônica 


\section{A. Tratamento LE na $1^{\text {a }}$ época}

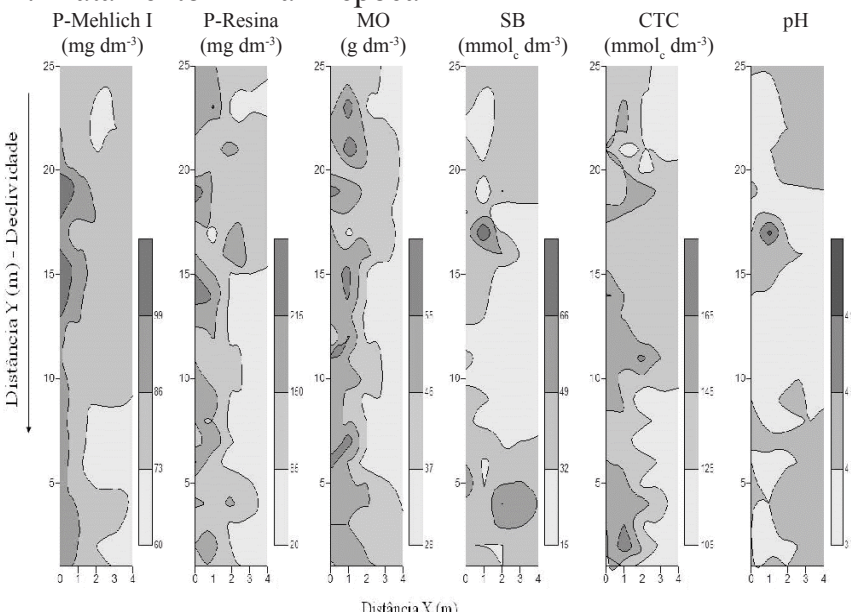

B. Tratamento LE na 2a época

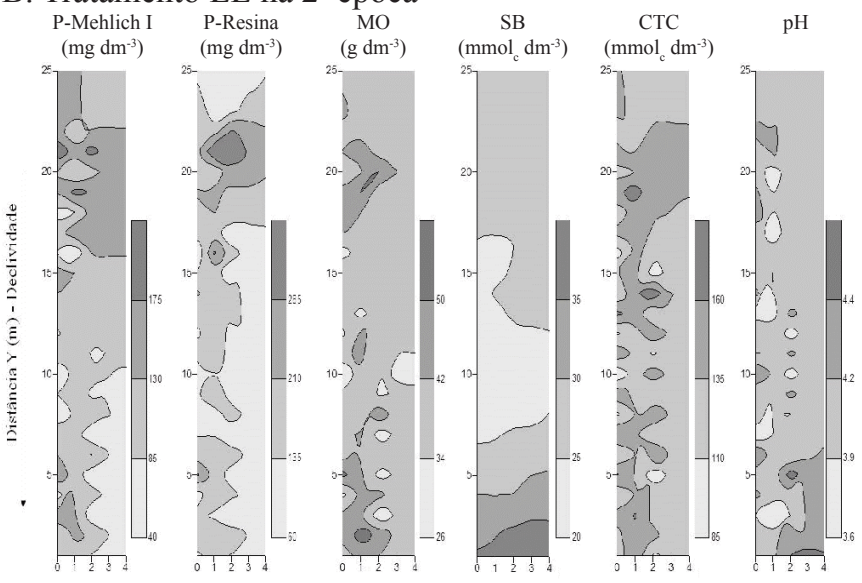

DistânciaX $(\mathrm{m})$

Figura 2. Mapas dos atributos químicos do solo para o tratamento lodo de esgoto (LE) na $1^{\text {a }}(\mathrm{A})$ e $2^{\mathrm{a}}(\mathrm{B})$ épocas estudadas. MO - Matéria orgânica. SB - Soma de bases. CTC - Capacidade de troca catiônica

A MO é apontada como causa da redução da adsorção de $\mathrm{P}$ no solo em razão do caráter aniônico dos produtos de sua decomposição (ácidos húmicos, fúlvicos, entre outros), os quais se ligam aos sítios de adsorção de $\mathrm{P}$ e aumentam a disponibilidade deste elemento no solo. A interação, quando ocorre, é por intermédio de cátions metálicos $\mathrm{Al}, \mathrm{Fe}, \mathrm{Ca}$ e $\mathrm{Mg}$ (Riggle \& Wandruszka, 2005). Este efeito foi sugerido por Guppy et al. (2005), ao observar que a adição de compostos derivados da mineralização da $\mathrm{MO}$ e $\mathrm{P}$ em solos oxídicos aumentou a adsorção deste elemento após seis dias de incubação. $\mathrm{O}$ aumento da adsorção de $\mathrm{P}$ pode ser, ainda, devido ao aumento da força iônica da solução como resultado das altas concentrações de carbono orgânico dissolvido. Além disto, a MO melhora a agregação das partículas do solo o que aumenta sua estabilidade contribuindo para reduzir o processo erosivo (Maria et al., 2007; Galdos et al., 2009). É provável que essas sejam as razões pelas quais a distribuição de $\mathrm{P}$ no solo tratado com lodo de esgoto tenha sido mais heterogênea já que não foram identificadas dependência espacial ou diferenças de concentrações ao longo do declive.
No tratamento adubação mineral (AM) todos os atributos avaliados revelaram dependência espacial entre os pontos amostrados, sendo o modelo esférico e o gaussiano os que proporcionaram melhor ajuste (Tabela 5). Da primeira para a segunda época o modelo para P-Resina se alterou de gaussiano para esférico. Alterações no modelo de ajuste podem ser reflexo das mudanças de comportamento das variáveis entre as épocas estudadas, quando a distribuição espacial do P-Resina se tornou menos estável.

Tabela 5. Parâmetros de ajuste dos semivariogramas dos atributos do solo no tratamento com adubação mineral (AM) no início do desenvolvimento da cultura do milho ( $1^{\text {a }}$ época) e após a colheita ( $2^{\mathrm{a}}$ época)

\begin{tabular}{|c|c|c|c|c|c|}
\hline Variável & $\mathrm{Co}_{0}$ & C & $a(m)$ & & $\mathrm{GD}\left[\mathrm{C}_{0} /\left(\mathrm{C}_{0}+\mathrm{C}\right)\right] * 100$ \\
\hline & \multicolumn{5}{|c|}{$1^{a}$ época } \\
\hline P-Mehlich I (mg dm-3) & 224 & 200 & 2,5 & Esférico & $53 \%$, Moderada \\
\hline P-Resina $\left(\mathrm{mg} \mathrm{dm}^{-3}\right)$ & 0,45 & 1,12 & 14,0 & Gaussiano & 29\%, Moderada \\
\hline $\mathrm{MO}\left(\mathrm{g} \mathrm{dm}^{-3}\right)$ & 1,1 & 1,2 & 8,8 & Esférico & $48 \%$, Moderada \\
\hline $\mathrm{SB}\left(\mathrm{mmol}_{\mathrm{c}} \mathrm{dm}^{-3}\right)$ & 310 & 328 & 12,5 & Gaussiano & $48 \%$, Moderada \\
\hline CTC $\left(\mathrm{mmol}_{\mathrm{c}} \mathrm{dm}^{-3}\right)$ & 0,77 & 0,45 & 12,3 & Gaussiano & $63 \%$, Moderada \\
\hline \multirow[t]{2}{*}{$\mathrm{pH}$} & 0,06 & 0,09 & 8,4 & Gaussiano & $40 \%$, Moderada \\
\hline & \multicolumn{5}{|c|}{$2^{a}$ época } \\
\hline P-Mehlich I (mg dm-3) & 0,79 & 0,34 & 8,0 & Esférico & $70 \%$, Moderada \\
\hline P-Resina $\left(\mathrm{mg} \mathrm{dm}^{-3}\right)$ & 400 & 269 & 13,0 & Esférico & $60 \%$, Moderada \\
\hline $\mathrm{MO}\left(\mathrm{g} \mathrm{dm}^{-3}\right)$ & 1,4 & 0,9 & 5,0 & Esférico & $61 \%$, Moderada \\
\hline $\mathrm{SB}\left(\mathrm{mmol}_{\mathrm{c}} \mathrm{dm}^{-3}\right)$ & 130 & 247 & 12,8 & Gaussiano & $34 \%$, Moderada \\
\hline CTC $\left(\mathrm{mmol}_{\mathrm{c}} \mathrm{dm}^{-3}\right)$ & 120 & 183 & 14,4 & Gaussiano & $40 \%$, Moderada \\
\hline $\mathrm{pH}$ & 0,04 & 0,05 & 8,5 & Gaussiano & 44\%, Moderada \\
\hline
\end{tabular}

MO - Matéria orgânica. SB - Soma de bases. CTC - Capacidade de troca de cátions. Co - Efeito pepita. C - Variância espacial. a - Alcance. GD - Grau de dependência espacial

O grau de dependência (GD) dos atributos do solo (Tabela 5) é determinado pela razão entre o efeito pepita $\left(\mathrm{C}_{0}\right)$ e o patamar $\left(\mathrm{C}_{0}+\mathrm{C}\right)$. Quanto maior o valor desta relação mais fraca é a dependência espacial dos atributos, ou seja, menor será a semelhança entre os valores vizinhos e a continuidade do fenômeno e, portanto, maior a variação de um ponto para outro (Vieira, 1997). Todas as variáveis apresentaram dependência espacial moderada pois apresentaram valores de GD entre 26 e 75\% (Cambardella et al., 1994).

No tratamento com aplicação de lodo de esgoto (LE) somente o atributo P-Mehlich I na primeira época e a soma de bases (SB) na segunda época apresentaram dependência espacial, com alcances de 2,4 e 11,0 m e graus de dependência moderados de 40 e $67 \%$, respectivamente (Tabela 6). Os atributos $\mathrm{P}$-Resina e $\mathrm{MO}$ na primeira e na segunda épocas e $\mathrm{SB}$, CTC e pH na segunda época, apresentaram efeito pepita puro, ou seja, ausência de dependência espacial.

Os semivariogramas das variáveis $\mathrm{SB}, \mathrm{CTC}$ e $\mathrm{pH}$ na primeira época sugeriram uma estrutura de bipartição (Tabela 6). Mesmo a transformação logarítmica dos dados para SB, variável que não demonstrou distribuição normal, não modificou o semivariograma. Tal efeito pode denotar a ocorrência de populações distintas na área as quais apresentam comportamentos diversos para diferentes distâncias, caso em que a semivariância dos atributos seria avaliada pela média das semivariâncias das populações, porém o número 
Tabela 6. Parâmetros de ajuste dos semivariogramas dos atributos do solo no tratamento com lodo de esgoto (LE) no início do desenvolvimento da cultura do milho ( $1^{\text {a }}$ época) e após a colheita ( $2^{\mathrm{a}}$ época)

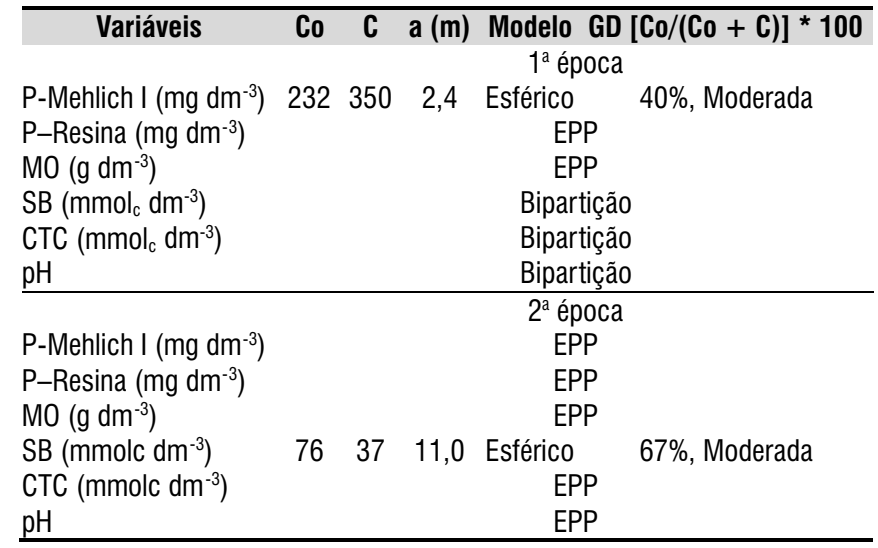

MO - Matéria orgânica. SB - Soma de bases. CTC - Capacidade de troca de cátions. Co - Efeito pepita. C - Variância espacial. a - Alcance. GD - Grau de dependência espacial. EPP - Efeito pepita puro

relativamente pequeno de amostras coletadas é inadequado para tal procedimento.

A ausência de dependência espacial para a maioria das variáveis no tratamento LE, está relacionada, provavelmente, à forma de disposição do lodo na parcela. Foram colocadas porções ao acaso sobre a superfície para posterior incorporação, que resultaram em pontos com acúmulo do lodo aplicado distribuídos de maneira aleatória.

$\mathrm{Na}$ segunda época a variável P-Mehlich I, que na $1^{\text {a }}$ época havia apresentado correlação espacial, mostrou ausência de dependência espacial (Tabela 6). Para esta variável observouse um pequeno aumento da semivariância até a distância de 10 $\mathrm{m}$ com dispersão infinita para distâncias superiores, o mesmo verificado para P-Resina e MO. Com a remoção da tendência sugerida por Vieira (2000), o resultado foi efeito pepita puro.

Determinadas as dependências espaciais das amostras foram construídos mapas utilizando-se interpolação por meio da krigagem. Para as amostras que não apresentaram dependência espacial utilizou-se o método de interpolação linear para interpolação dos valores. A distribuição espacial do $\mathrm{P}$ e dos demais atributos químicos do solo na área estudada nos tratamentos AM e LE nas duas épocas, está apresentada nas Figuras 1 e 2, respectivamente.

No tratamento AM (Figura 1) observou-se aumento dos teores de P-Mehlich I, SB, CTC, $\mathrm{pH}$ e, principalmente, $\mathrm{P}$-Resina e MO na parte inferior da parcela, nos dois períodos de amostragem, indicando que a declividade e a ação da erosão hídrica contribuíram para o carreamento dos nutrientes via enxurrada na direção do declive, acumulando-os na porção inferior da parcela. No tratamento LE (Figura 2) observamse áreas com concentrações mais elevadas e outras com concentrações mais baixas dos atributos estudados ao longo das parcelas, tanto no início ( $1^{\text {a }}$ época) como no final do ciclo da cultura ( $2^{a}$ época).

Os mapas expressam as diferenças entre os dados com distribuição aleatória e com dependência espacial. Os atributos que permitiram ajuste do semivariograma e foram espacializados por krigagem (P-Mehlich I para a primeira época e SB para a segunda época) apresentam áreas contíguas de mesma classe de valores dos atributos. Os atributos com distribuição aleatória geraram mapas com distribuição irregular de áreas de diferentes classes de valores, de modo que pontos próximos entre si apresentassem concentrações bastante distintas, exprimindo o efeito pepita dos semivariogramas neste tratamento.

De modo geral, tais resultados rejeitam a suposição de que a aplicação de lodo de esgoto, em comparação com a adubação mineral, ocasionaria maior arraste de $\mathrm{P}$ associado às partículas do solo e do próprio lodo para a parte inferior das parcelas. Este maior teor de P no solo poderia resultar em aumento no $P$ arrastado pela enxurrada nos tratamentos com lodo comprometendo, desta forma, a qualidade ambiental por aumentar os riscos de eutrofização. Os dados são confirmados pelos resultados obtidos por Costa et al. (2010), que observaram que altos teores de $\mathrm{P}$ no solo que recebeu lodo não contribuíram para aumentar seu transporte.

\section{Conclusões}

1. A aplicação de lodo de esgoto no solo feita de maneira não uniforme, resulta em sítios com maiores teores de fósforo aleatoriamente distribuídos.

2. O lodo de esgoto aumenta os teores de P disponível no solo porém não promove maior arraste desse nutriente para as porções inferiores das parcelas.

3. A adubação mineral resulta em maiores quantidades de $\mathrm{P}$ na parte inferior da área agrícola pelo arraste ocasionado pela erosão. $\mathrm{O}$ mesmo padrão de distribuição espacial ocorre para $\mathrm{SB}, \mathrm{CTC}$, $\mathrm{pH}$ e $\mathrm{MO}$.

4. O fósforo disponível no solo está diretamente correlacionado com o teor de matéria orgânica quando é feita aplicação de lodo de esgoto.

\section{Literatura Citada}

Bettiol, W.; Ghini, R. Impacts of sewage sludge in tropical soil: A case study in Brazil. Applied and Environmental Soil Science, v.201, p.1-11, 2011.

Boeira, R. C.; Souza, M. D. Estoque de carbono orgânico e de nitrogênio, $\mathrm{pH}$ e densidade de um Latossolo após três aplicações de lodos de esgoto. Revista Brasileira de Ciência do Solo, v.31, p.581-590, 2007.

Camargo, L. A.; Marques Júnior, J.; Pereira, G. T.; Alleoni, L. R. F. Spatial correlation between the composition of the clay fraction and contents of available phosphorus of an Oxisol at hillslope scale. Catena, v.100, p.100-106, 2012.

Camargo, O. A.; Moniz, A. C.; Jorge, J. A.; Valadares, J. M. A. S. Métodos de análise química, mineralógica e física de solos do Instituto Agronômico de Campinas. Campinas: Instituto Agronômico, 1986. 94p. Boletim Técnico, n.106. 
Cambardella, C. A.; Moorman, T. B.; Novak, J. M.; Parkin, T. B.; Karlen, D. L.; Turco, R. F.; Konopka, A. E. Field-Scale variability of soil properties in central Iowa soils. Soil Science Society of America Journal, v.58, p.1501-1511, 1994.

CETESB - Companhia de Tecnologia de Saneamento Ambiental. Aplicação de biossólidos de sistemas de tratamento biológico em áreas agrícolas. Critérios para projeto e operação: Manual técnico. Norma P430. São Paulo: CETESB. 1999. 33p.

Costa, V. L.; Maria, I. C. de; Camargo, O. A. Transporte de fósforo pela enxurrada em Latossolo que recebeu lodo de esgoto. Bragantia, v.69, p.115-123, 2010.

Elliott, H. A.; O'Connor, G. A. Phosphorus management for sustainable biosolids recycling in the United States. Soil Biology \& Biochemistry, v.39, p.1318-1327, 2007.

EMBRAPA - Empresa Brasileira de Pesquisa Agropecuária. Centro Nacional de Pesquisa de Solos. Sistema brasileiro de classificação de solos. 2.ed. Rio de Janeiro: Embrapa Solos, 2006. 306p.

Galdos, M. V.; Maria, I. C. de; Camargo, O. A.; Dechen, S. C. F. Sewage sludge application on cultivated soils: effects on runoffand trace metal load. Scientia Agricola, v.66, p.368376, 2009.

Guppy, C. N.; Menzies, N. W.; Blamey, F. P. C.; Moody, P. W. Do decomposing organic matter residues reduce phosphorus sorption in highly weathered soils? Soil Science Society of America Journal, v.69, p.1405-1411, 2005.

Hart, M. R.; Quin, B. F.; Nguyen, M. L. Phosphorus runoff from agricultural land direct fertilizer effects: a review. Journal of Environmental Quality, v.33, p.1954-1972, 2004.

Haygarth, P. M.; Jarvis, S. C. Transfer of phosphorus from agricultural soils. Advances in Agronomy, v.66, p.195-219, 1999.

Kidd, P. S.; Dominguez-Rodriguez, M. J.; Diez, J.; Monterroso, C. Bioavailability and plant accumulation of heavy metals and phosphorus in agricultural soils amended by long-term application of sewage sludge. Chemosphere, v.66, p.14581467, 2007.

Maria, I. C.; Kocssis, M. A.; Dechen, S. C. F. Agregação do solo em área que recebeu lodo de esgoto. Bragantia, v.66, p.291-298, 2007.

Mori, H. F.; Favaretto, N.; Pauletti, V.; Dieckow, J.; Santos, W. L. Perda de água, solo e fósforo com aplicação de dejeto líquido bovino em Latossolo sob plantio direto e com chuva simulada. Revista Brasileira de Ciência do Solo, v.33, p.189-198, 2009.
Munhoz, R. O.; Berton, R. S.; Camargo, O. A. Phosphorus sorption and redistribution on soil solid phase in a brazilian haplorthox amended with biosolids. Applied and Environmental Soil Science, v.2011, p.1-7, 2011.

Novais, R. F.; Smyth, T. J.; Nunes, F. N. Fósforo. In: Novais, R. F.; Alvarez V., V. H.; Barros, N. F.; Fontes, R. L. F.; Cantarutti, R. B.; Neves, J. C. L. Fertilidade do solo. Viçosa: SBCS, 2007. p.471-550.

Raij, B. van; Andrade, J. C.; Cantarella, H.; Quaggio, J. A. (ed.) Análise química para avaliação da fertilidade de solos tropicais. Campinas: Instituto Agronômico, 2001. 285p.

Raij, B. van; Cantarella, H.; Quaggio, A. J.; Furlani, A. M. C. Recomendações de adubação e calagem para o Estado de São Paulo. 2.ed. Campinas: Instituto Agronômico/Fundação IAC, 1996. 285p.

Riggle, J.; Wandruszka, R. von. Binding of inorganic phosphate to dissolved metal humates. Talanta, v.66, p.372-375, 2005.

Shen, J. B.; Yuan, L. X.; Zhang, J. L.; Li, H. G.; Bai, Z. H.; Chen, X. P.; Zhang, W. F. Zhang, F.S. Phosphorus dynamics: from soil to plant. Plant Physiology, v.156, p.997-1005, 2011.

Silva, F. C. Manual de análises químicas de solos, plantas e fertilizantes. Brasília: EMBRAPA, 1999. 370p.

Souza, D. M. G.; Miranda, L. N.; Oliveira, S. A. Acidez do solo e sua correção. In: Novais, R. F.; Alvarez V., V. H.; Barros, N. F.; Fontes, R. L. F.; Cantarutti, R. B.; Neves, J. C. L. Fertilidade do solo. Viçosa: SBCS, 2007a. p.205-274.

Souza, Z. M.; Barbiere, D. M.; Jr. Marques, J.; Pereira, J. T.; Campos, M. C. C. Influencia da variabilidade espacial de atributos químicos de um Latossolo na aplicação de insumos para cultura de cana-de-açúcar. Ciência \& Agrotecnologia, v.31, p.371-377, 2007b.

Trannim, I. C. B.; Siqueira, J. O.; Moreira, F. M. S. Atributos químicos e físicos de um solo tratado com biossólido industrial e cultivado com milho. Revista Brasileira de Engenharia Agrícola e Ambiental, v.12, p.223-230, 2008.

Vieira, S. R. Variabilidade espacial de argila, silte e atributos químicos em uma parcela experimental de um Latossolo Roxo de Campinas (SP). Bragantia, v.56, p.181-190, 1997.

Vieira, S. R. Geoestatística em estudos de variabilidade espacial do solo. In: Novais, R. F.; Alvares, V. H.; Schaefer, C. E. G. R. Tópicos em ciência do solo. Viçosa: Sociedade Brasileira de Ciência do Solo, v.1, 2000. p.1-53.

Vieira, S. R.; Millete, J.; Topp, G. C.; Reynolds, W. D. Handbook for geostatistical analysis of variability in soil and climate data. In: Alvarez, V. V. H.; Schaefer, C. E. G. R.; Barros, N. F.; Mello, J. W. V.; Costa, L. M. (ed.). Tópicos em ciência do solo. Viçosa: Sociedade Brasileira de Ciência do Solo, v.2, 2002.45p. 